\title{
Downregulation of IncRNA H19 alleviates atherosclerosis through inducing the apoptosis of vascular smooth muscle cells
}

\author{
HUI SUN ${ }^{1 *}$, QIANQIAN JIANG ${ }^{2 *}$, LI SHENG ${ }^{1}$ and KAI CUI ${ }^{3}$ \\ ${ }^{1}$ Department of Cardiology, Jining First People's Hospital, Jining, Shandong 272100; ${ }^{2}$ Department of Cardiology, \\ The Second People's Hospital of Liaocheng, Linqing, Liaocheng, Shandong $252601{ }^{3}$ Geriatrics Department, \\ The Central Hospital of Shengli Oilfield, Dongying, Shandong 257034, P.R. China
}

Received May 1, 2019; Accepted October 30, 2019

DOI: $10.3892 / \mathrm{mmr} .2020 .11394$

\begin{abstract}
Aberrant proliferation and apoptosis of vascular smooth muscle cells (VSMCs) serve a dominant role in the pathogenesis of atherosclerosis (AS). Long non-coding (lnc) RNA H19 is reported to accelerate the progression of AS by inhibiting the apoptosis of VSMCs, whereas p53 is identified as promoting VSMC apoptosis. The present study aimed to explore the effects of $\mathrm{H} 19 / \mathrm{p} 53$ on the pathogenesis of AS. Apolipoprotein E knockout $\left(\mathrm{ApoE}^{-/-}\right)$mice fed a high-fat diet were used as in vivo AS models. Reverse transcription-quantitative PCR and western blot were used to detect mRNA and protein expression levels, respectively. VSMC proliferation and apoptosis were respectively assessed by CCK- 8 and flow cytometry. Compared with the control group, mouse weight and plaque area were all increased in the AS model group, as was the expression of H19. Knockdown of H19 reduced the proliferation and induced apoptosis of VSMCs, and increased the expression of p53, cleaved caspase3 (c-caspase3) and p53 upregulated modulator of apoptosis, as well as enhancing the interaction between Bax and p53 proteins. Downregulation of $\mathrm{H} 19$ reduced the plaque area and promoted the expression of c-caspase 3 in mouse aortic tissues in vivo, as well as enhancing the effects of simvastatin, a drug used for AS treatment. Results from the present study indicated that knockdown of $\mathrm{H} 19$ may prevent AS deterioration through increased p53-mediated VSMC apoptosis.
\end{abstract}

\section{Introduction}

Atherosclerosis (AS) is a chronic multi-factorial vascular disease and an underlying cause of cardiovascular disease,

Correspondence to: Dr Kai Cui, Geriatrics Department, The Central Hospital of Shengli Oilfield, 31 Jinan Road, Dongying, Shandong 257034, P.R. China

E-mail:kaicui@163.com

*Contributed equally

Key words: long non-coding RNA H19, p53, cell apoptosis, drug sensitivity, atherosclerosis which accounts for 16.7 million deaths each year worldwide (1). AS is a major risk factor for cardiovascular and cerebrovascular diseases (2) and is characterized by lipid accumulation and the formation of fat-laden plaque in vessels (3). Despite advances in treatment, the morbidity and mortality of AS remain at high levels. Therefore, it is urgent to uncover new mechanisms underlying atherogenesis and to find potent therapeutic targets and methods for AS.

The pathogenesis of AS is a complex process, in which the dysfunction of vascular smooth muscle cells (VSMCs) and endothelial cells, as well as the excessive secretion of pro-inflammatory cytokines by macrophages serve important roles $(4,5)$. Particularly, VSMCs serve a crucial role in this process owing to their capacities to promote plaque growth in early stages and to contribute to plaque stability in advanced stages (6), which suggested that VSMCs may be an efficient therapeutic target for AS.

Although $\sim 90 \%$ of the human genes are transcribed, only $2 \%$ of the human genome possesses the ability to encode proteins, the remaining being non-coding genes $(7,8)$. Long non-coding (lnc)RNAs are a class of ncRNAs that have been demonstrated to regulate gene expression at transcription and post-transcription levels (9). Accumulated evidence has shown that lncRNAs are involved in a number of pathological processes, including carcinogenesis (10) and chronic diseases such as AS (11-13). IncRNA H19 (H19) is transcribed from the H19/insulin-like growth factor 2 gene cluster that is located in human chromosome 11p15.5 (14). Recently, H19 was reported to be highly expressed in patients with AS and upregulation of H19 promoted the proliferation and repressed the apoptosis of VSMCs and human umbilical vein endothelial cells (HUVECs) (15). However, the detailed mechanisms underlying H19 in AS remains to be elucidated.

p53 is an essential molecule in the regulation of cell growth, apoptosis and cell cycle, and it is thought to be strongly implicated in the pathogenesis of AS $(16,17)$. The inactivation of p53 promotes the development of AS (18-20). Wu et al (21) demonstrated that long intergenic non-coding RNA-p21 (lincRNA-p21) represses proliferation and promotes apoptosis of VSMCs by increasing p53 expression. However, whether p53 is involved in H19-regulated VMSC proliferation and apoptosis remains unclear. Therefore, the present study aimed to explore the effects of $\mathrm{H} 19 / \mathrm{p} 53$ in regulating VMSCs 
apoptosis and proliferation in vitro and in vivo, thereby elucidating its role in the pathogenesis of AS.

\section{Materials and methods}

Ethics approval. Animal experiments were approved by the Committee of Jining First People's Hospital (Jining, China) and was performed in accordance with the National Institutes of Health Guide for the Care and Use of Laboratory Animals.

Animal experiments. Male specific pathogen free (SPF) grade apolipoprotein (Apo) $\mathrm{E}^{-/-}$mice $(\mathrm{n}=25$; age, 8 weeks; weight, 20-22 g) were purchased from Genomeditech and were used to establish AS models. Wild-type C57BL/6J mice without ApoE knockdown ( $\mathrm{n}=5$; age, 8 weeks; weight, 20-22 g) were used as the control (control group). All mice were raised under SPF conditions in a controlled temperature $\left(23 \pm 2{ }^{\circ} \mathrm{C}\right)$ and humidity (55-60\%) environment under a $12 \mathrm{~h}$ light/dark cycle. After 1 week of acclimation, the $\mathrm{ApoE}^{-/-}$mice $(n=25)$ were divided into five groups ( $n=5$ mice/group): i) AS group; ii) $\mathrm{AS}+$ short hairpin RNA (sh)-negative control (NC) group; iii) $\mathrm{AS}+$ sh-H19 group; iv) $\mathrm{AS}+$ sh-NC + simvastatin group; and v) AS + sh-H19 + simvastatin group. To induce AS models, the $\mathrm{ApoE}^{-/-}$mice were given a high-fat diet comprising $21 \%$ fat and $0.15 \%$ cholesterol on the basis of a chow diet for 8 weeks as in a previous study (22). Following 8 weeks of receiving the high-fat diet, the mice were subsequently fed a standard laboratory diet (10\% fat, $15 \%$ protein and $75 \%$ carbohydrate) for the remaining 10 weeks. Mice in the control group were fed a standard laboratory diet for 18 weeks. Mice in the $\mathrm{AS}+$ sh-H19 group were given intraperitoneal injection of $100 \mu 1$ shRNA targeting mouse H19 (sh-H19) once/week for 10 consecutive weeks after high-fat diet administration, and the lentiviral vector sh-NC was used as a negative control of sh-H19. Additionally, the AS + sh-NC + simvastatin group and the AS + sh-H19 + simvastatin group were given an oral administration of $5 \mathrm{mg} / \mathrm{kg}$ of simvastatin (Merck KGaA) every day for 10 weeks following 8 weeks of high-fat diet administration (23), whereas mice in other groups were orally administrated with vehicle (80\% Tween).

Thoracic aorta collection and histopathological analysis. At the end of sh-H19 and simvastatin administration, mice were sacrificed by cervical dislocation and the entire thoracic aortas (from the branches of the abdominal aorta to the aortic arch) were removed and stored in liquid nitrogen for pathological examination or RNA extraction. For histopathological analysis, the aortic arch was fixed with $10 \%$ formaldehyde at room temperature for $24 \mathrm{~h}$. Subsequently, samples were dehydrated with different concentrations $(80,95$ and $100 \%)$ of ethyl alcohol, cleared with xylene, embedded in paraffin and sectioned $(5 \mu \mathrm{m})$. Sections were stained with $100 \%$ hematoxylin (Beyotime Institute of Biotechnology) for $3 \mathrm{~min}$ and $0.5 \%$ eosin (Beyotime Institute of Biotechnology) for $30 \mathrm{sec}$ at room temperature. The staining was visualized using an inverted light microscope at a magnification of x 100 .

Immunohistochemical staining. Tissue sections were deparaffinized and rehydrated with xylene and ethanol, as aforementioned. Subsequently, the antigen was retrieved using citrate antigen retrieval solution (Beyotime Institute of Biotechnology) and blocked with 5\% goat serum (AmyJet Scientific, Inc.) diluted in TBS $+0.5 \%$ Tween 20 (TBST), the sections were probed with the primary antibody against cleaved caspase 3 (c-caspase3; 1:100; cat. no. 9661; Cell Signaling Technology, Inc.) at $4^{\circ} \mathrm{C}$ overnight. Following the primary antibody incubation, sections were incubated with a horseradish peroxidase-conjugated secondary antibody (1:500; cat. no. 18653; Cell Signaling Technology, Inc.) at room temperature for $1 \mathrm{~h}$, followed by incubation with the chromogen 3,30-diaminobenzidine tetrachloride ( $\&$ \& Systems, Inc.) for 2-3 sec at room temperature. Cell nuclei were stained with $1 \%$ Harris hematoxylin solution for $30 \mathrm{sec}$ at room temperature. The staining of c-caspase 3 was evaluated by two pathologists on the basis of the positive staining proportion and the staining intensity (24). The positive staining percentage was scored as 0 for $\leq 5 \%, 1$ for $6-25 \%, 2$ for $26-50 \%, 3$ for $51-75 \%$ and 4 for $\geq 75 \%$, respectively. Intensity was marked as follows: 0 , no staining; 1 weak staining; 2 , moderate staining; and 3 , strong staining. The final score was obtained by multiplying the percentage score and intensity score.

Cell culture and simvastatin treatment. Human VSMCs (ATCC CRL-1999) were obtained from the American Type Culture Collection. VSMCs were cultured in DMEM (HyClone; GE Healthcare Life Sciences), supplemented with $1 \%$ penicillin $(100 \mathrm{U} / \mathrm{ml}) /$ streptomycin $(100 \mathrm{mg} / \mathrm{ml})$ (Beyotime Institute of Biotechnology) and 10\% FBS (Gibco; Thermo Fisher Scientific, Inc.) and maintained in a $37^{\circ} \mathrm{C}$ constant humidified incubator with $5 \% \mathrm{CO}_{2}$.

For simvastatin treatment, a total of $5 \times 10^{5} \mathrm{VSMCs} / \mathrm{ml}$ were incubated with $2 \mu \mathrm{M}$ simvastatin (Xingqiong Co., Ltd.) for $24 \mathrm{~h}$ following transduction for $24 \mathrm{~h}$ with the lentiviral vectors. H19 and p53 expression levels were reduced by transfecting cells with $2 \mathrm{mg}$ shRNAs (lentiviral vectors) targeting H19 (sh-H19) and p53 (sh-p53), respectively, and control cells were transduced with $2 \mu \mathrm{g}$ negative control vector (sh-NC); all shRNAs were purchased from Shanghai GenePharma Co., Ltd. VSMCs were transduced with sh-p53, sh-H19 or sh-NC in a pGLVH1/GFP+ Puro lentiviral vector (Shanghai GenePharma Co., Ltd.) using Polybrene (4 $\mu \mathrm{g} / \mathrm{ml}$; Merck KGaA).

Reverse transcription-quantitative PCR (RT-qPCR). The expression levels of $\mathrm{H} 19 / \mathrm{p} 53$ in mouse aorta and VSMCs were tested by RT-qPCR; expression levels were normalized to GAPDH. Briefly, total RNA was acquired from cells $\left(1 \times 10^{6}\right.$ cells/sample) and aorta tissues (100 g/sample) by using TRIzol $^{\circledR}$ reagent (Invitrogen; Thermo Fisher Scientific, Inc.), according to the manufacturer's protocol. Subsequently, $1 \mu \mathrm{g}$ of the total RNA was reversed transcribed using a cDNA Synthesis Kit (CoWin Biosciences), according to the manufacturers' protocol. qPCR was performed using the $S Y B^{\circledR}$ Green PCR Master mix (CoWin Biosciences), according to the manufacturer's protocol using the following primers: H19, forward 5'-ATCGGTGCCTCAGCGTTCGG-3', reverse 5'-CTGTCCTCGCCGTCACACCG-3'; p53, forward 5'-CCT GGATTGGCCAGACTGC-3', reverse 5'-TTTTCAGGA AGTAGTTTCCATAGGT-3'; GAPDH, forward 5'-CACTAG GCGCTCACTGTTCTCT-3', reverse 5'- CGTTCTCAGCCT TGACGGT-3'. The following thermocycling conditions for 
the qPCR were used: Initial denaturation for $3 \mathrm{~min}$ at $95^{\circ} \mathrm{C}$; followed by 35 cycles of denaturation at $95^{\circ} \mathrm{C}$ for $10 \mathrm{sec}$ and annealing/extension at $55^{\circ} \mathrm{C}$ for 30 . Expression levels were quantified using the $2^{-\Delta \Delta \mathrm{Cq}}$ method (25) and normalized to GAPDH.

Western blot analysis. VMSCs were collected and lysed with RIPA lysis buffer (cat. no. R0010; Beijing Solarbio Science \& Technology Co., Ltd.) containing protease inhibitor. After being centrifuged at $20,000 \mathrm{x}$ g for $30 \mathrm{~min}$ at $4^{\circ} \mathrm{C}$ and quantified with a BCA kit (Thermo Fisher Scientific, Inc.), $30 \mu \mathrm{g}$ protein from each sample was separated by $10 \%$ SDS-PAGE and transferred onto PVDF membranes (EMD Millipore). The membranes were blocked with 5\% nonfat milk diluted in TBST at room temperature for $1 \mathrm{~h}$ and then incubated with anti-p53 (1:1,000; cat. no. 9282; Cell Signaling Technology, Inc.), anti-bcl-2 (1:1,000; cat. no. 3498; Cell Signaling Technology, Inc.), anti-p53 upregulated modulator of apoptosis (PUMA; 1:1,000; cat no. 4976; Cell Signaling Technology, Inc.), anti-c-caspase3 (1:2,000; cat. no. 9661; Cell Signaling Technology, Inc.) and anti-GAPDH monoclonal antibody (1:5,000; cat. no. 5174; Cell Signaling Technology, Inc.) at $4^{\circ} \mathrm{C}$ overnight. After washing with TBST three times, the membranes were probed with the horseradish peroxidase-conjugated goat anti-mouse and goat anti-rabbit secondary antibodies $(1: 10,000$; cat. nos. SA00001-1 and SA00001-2, respectively; ProteinTech Group, Inc.) for $1 \mathrm{~h}$ at room temperature and visualized with ECL detection reagents (EMD Millipore). Protein expression was quantified using ImageJ version 1.48 software (National Institutes of Health). GAPDH was used as a loading control to normalize the data.

Co-immunoprecipitation analysis of the interaction of p53 and Bax proteins. VSMCs transfected with sh-H19 or sh-NC were collected and rinsed with cold PBS and then lysed in IP Lysis Buffer (Thermo Fisher Scientific, Inc.), followed by centrifugation at $20,000 \times \mathrm{g}$ for $30 \mathrm{~min}$ at $4^{\circ} \mathrm{C}$. Next, total protein was quantified using a BCA assay and the lysate containing $200 \mu \mathrm{g}$ protein were incubated with Dynabeads ${ }^{\circledR}$ protein $\mathrm{G}$ for $1 \mathrm{~h}$ and incubated with $2 \mu \mathrm{g}$ anti-p53 (cat. no. 9282; Cell Signaling Technology, Inc.) or anti-Bax (cat. no. ab216494; Abcam) antibodies overnight at $4^{\circ} \mathrm{C}$, followed by incubation with Dynabeads ${ }^{\circledR}$ protein $\mathrm{G}$ for another $1 \mathrm{~h}$. The whole cell lysate was incubated overnight at $4^{\circ} \mathrm{C}$ with $2 \mu \mathrm{g}$ anti-rabbit IgG antibody (cat. no. 3900; Cell Signaling Technology, Inc.) as the negative control for the anti-p53 antibody or $2 \mu \mathrm{g}$ anti-mouse IgG antibody (cat. no. ab6709; Abcam) as the negative control for the anti-Bax antibody. The immunocomplex were washed with IP Lysis Buffer and analyzed by the aforementioned western blot procedure using anti-Bax (1:1,000; cat. no. 2774; Cell Signaling Technology, Inc.) or anti-p53 (1:1,000; cat. no. 2524; Cell Signaling Technology, Inc.) antibodies.

Cell proliferation detection. Cell proliferation was measured by Cell Counting Kit-8 (CCK-8; Dojindo Molecular Technologies, Inc.) according to the manufacturer's protocol. Briefly, $100 \mu \mathrm{l}$ cell suspension containing $2 \times 10^{3}$ cells was placed into a 96 -well plate and incubated at $37^{\circ} \mathrm{C}$ overnight. Then, the cells were transfected with sh-NC, sh-H19, sh-p53 or sh-H19 + sh-p53 for 1, 2, 3, 4 or 5 days, with the medium changed every day. At each time point, $10 \mu \mathrm{l}$ CCK- 8 solution was added to the wells and the absorbance at $450 \mathrm{~nm}$ was detected using a spectrophotometer (Shjingmi Co., Ltd.).

Cell apoptosis. Cell apoptosis was assessed by using Annexin V-FITC/PI Double-Staining kit (YESEN Co., Ltd; https://www.yeasen.com), according to the manufacturer's protocol. After staining, cells were analyzed using a BD FACSCalibur flow cytometer (BD Biosciences) at $488 \mathrm{~nm}$ excitation and $630 \mathrm{~nm}$ emission to determine apoptotic rates with FlowJo version 10 software (FlowJo LLC).

Statistical analysis. Data from three independent experiments are presented as the mean \pm SD. Comparisons were made using Student's t-test or with one-way ANOVA followed by Tukey's test if $>2$ groups using SPSS 17.0 software (SPSS, Inc.). $\mathrm{P}<0.05$ was considered to indicate a statistically significant difference.

\section{Results}

H19 is highly expressed in AS model mice and H19 downregulation promotes VSMC apoptosis. To further explore the effect of H19 in the pathogenesis of AS, the expression levels of H19 in the mouse AS model were assessed. The average mouse body weights following 8 weeks of high-fat diet were clearly increased in the AS model group compared with the control group (Fig. 1A). In addition, compared with the control group, plaque formation was visible in the aortic roots of mice in the AS group and demonstrated significantly increased lesion sizes (Fig. 1B), which indicated that the AS model had been constructed successfully. H19 expression patterns in mouse aorta tissues from the control and the AS group were compared using RT-qPCR. The results demonstrated that H19 expression levels were significantly higher in AS group mice compared with the control group (Fig. 1C).

The effects of H19 on the proliferation and apoptosis of VSMCs were investigated through loss-of-function experiments. The mRNA level of $\mathrm{H} 19$ was reduced by approximately $80 \%$ after VSMCs were infected with sh-H19 (Fig. 1D). Silencing of H19 significantly inhibited the viability (Fig. 1E) and promoted the apoptosis of VSMCs (Fig. 1F and G). These results suggested that $\mathrm{H} 19$ may serve an important role in the regulation of VSMC growth.

Downregulation of $\mathrm{H} 19$ induces the apoptosis of VSMCs through enhancing p53 expression. The role of p53 in H19 downregulation-induced VMSC apoptosis promotion was next investigated. The expression of p53 was significantly increased when H19 expression was reduced in VSMCs at both mRNA and protein levels (Fig. 2A and B), which was evidently rescued when p53 expression was silenced (Fig. 2C and D). p53 downregulation also impaired sh-H19 roles in inhibiting VSMC viability (Fig. 2E) and promoting VSMC apoptosis (Fig. 2F and G).

Downregulation of $\mathrm{H} 19$ in VSMCs enhanced the interaction between p53 protein and Bax protein (Fig. 3A). In addition, the expression levels of PUMA and c-caspase 3 were increased, whereas Bcl-2 expression was reduced in the sh-H19 group compared with the sh-NC group, and this effect was neutralized when p53 was also downregulated in VSMCs (Fig. 3B and C). Together, these findings indicated 
A
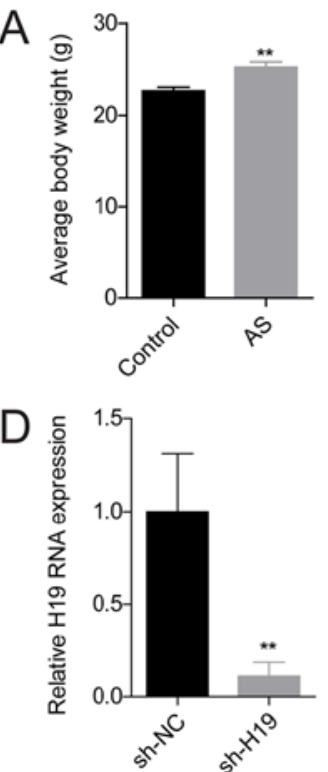

$\mathrm{B}$
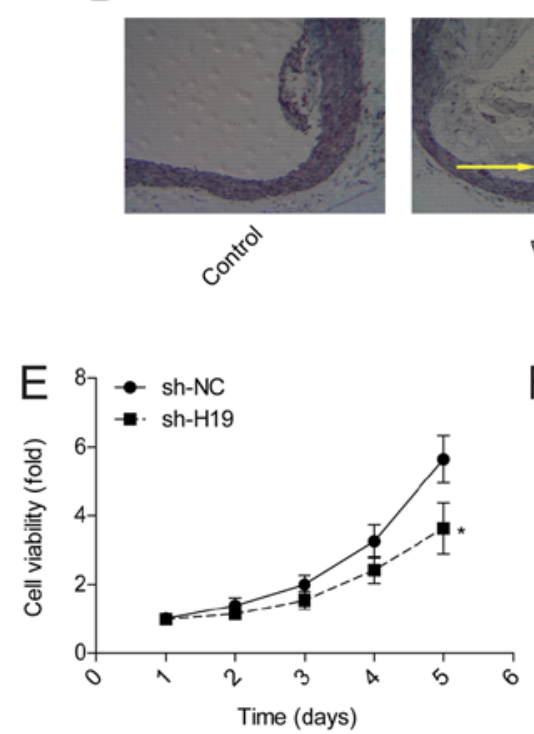

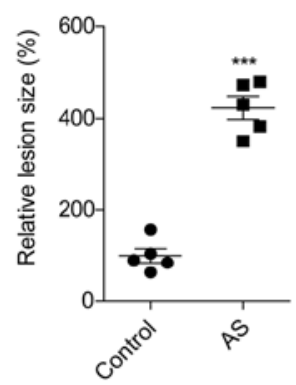

F

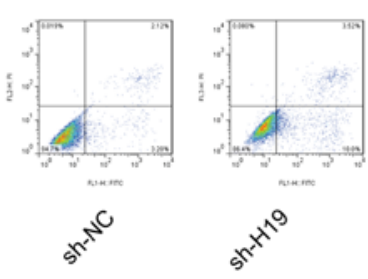

C

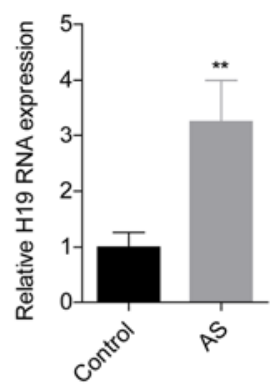

G

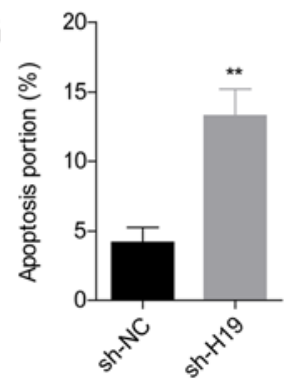

Figure 1. H19 is overexpressed in mouse AS models and downregulation of H19 inhibits proliferation and induces apoptosis of VSMCs. (A) Average body weight of mice in the control and AS groups. (B) Hematoxylin and eosin staining of the lesions in aortic roots of mice in the control and AS groups and the quantification of lesion size. Yellow arrow indicates atherosclerotic lesions. Magnification, x200. (C) RT-qPCR analysis of the mRNA expression levels of $\mathrm{H} 19$ in the aortic roots of mice in the control and AS groups. (D) RT-qPCR was used to test the knockdown efficiency of sh-H19 in VSMCs. (E) Cell viability was detected by Cell Counting Kit-8 assay after VSMCs were infected with sh-NC or sh-H19. (F and G) Cell apoptosis was measured by flow cytometry after VSMCs were infected with sh-NC or sh-H19. ${ }^{*} \mathrm{P}<0.05,{ }^{* *} \mathrm{P}<0.01$ and ${ }^{* * *} \mathrm{P}<0.001$ vs. Control or sh-NC. AS, atherosclerosis; NC, negative control; RT-qPCR, reverse transcription-qPCR; sh, short hairpin RNA; VSMCs, vascular smooth muscle cells.

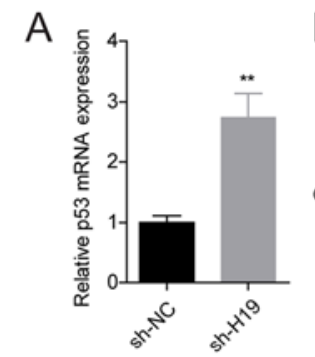

B

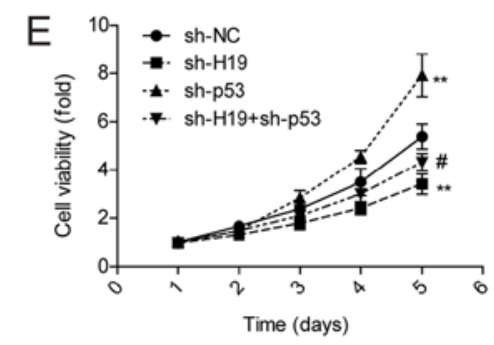

$\mathrm{F}$
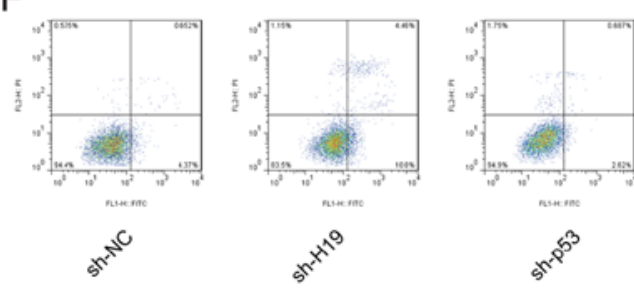
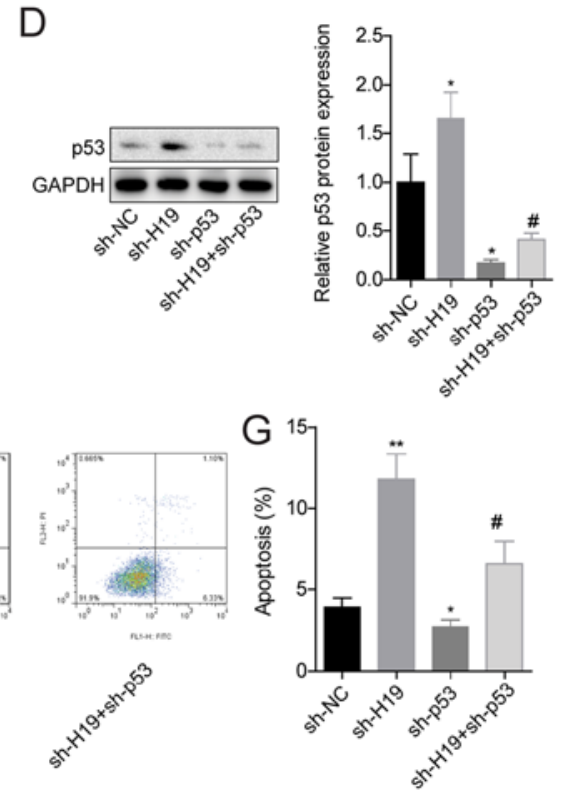

Figure 2. H19 downregulation promotes cell apoptosis through upregulating p53 expression in VSMCs. (A) mRNA and (B) protein expression levels of p53 were assessed by RT-qPCR and western blotting, respectively. (C) p53 mRNA expression levels after VSMCs were infected with sh-NC or sh-p53. (D) p53 protein expression of p53 was detected after VSMCs were infected with sh-NC, sh-H19, sh-53 or sh-H19+ sh-p53. (E) Cell proliferation was detected by Cell Counting Kit-8 assay after VSMCs were infected with sh-NC, sh-H19, sh-p53 and sh-H19+ sh-p53. (F and G) Cell apoptosis was measured by flow cytometry after VSMCs were infected with sh-NC, sh-H19, sh-p53 and sh-H19+ sh-p53. ${ }^{*} \mathrm{P}<0.05$ and ${ }^{* *} \mathrm{P}<0.01$ vs. sh-NC; ${ }^{*} \mathrm{P}<0.05$ vs. sh-H19. NC, negative control; RT-qPCR, reverse transcription-qPCR; sh, short hairpin RNA; VSMCs, vascular smooth muscle cells.

that H19 downregulation promoted VSMC apoptosis in a p53-dependent manner.

Knockdown of $H 19$ represses plaque formation in Apo $E^{-/-}$mice. Next, the effects of H19 downregulation in the pathogenesis of
AS in $\mathrm{ApoE}^{-/-}$mice was explored. Compared with the AS group, the lesion size in the aortic root was significantly reduced in AS + sh-H19 group (Fig. 4A). The downregulation of H19 in the AS + sh-H19 group significantly increased the expression of c-caspase 3 , an apoptotic marker in atherosclerotic plaque 
A

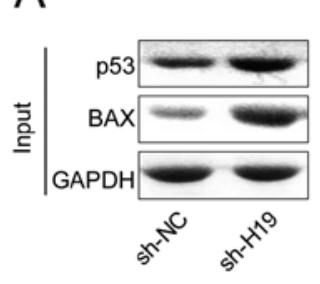

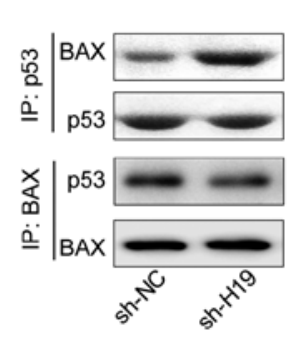

B

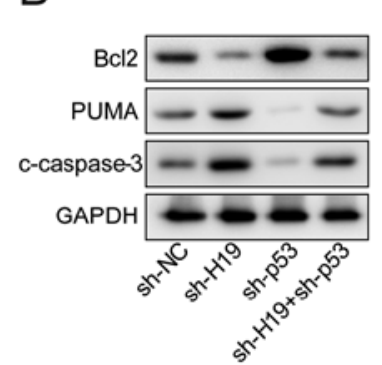

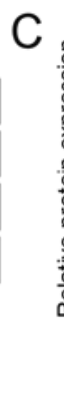

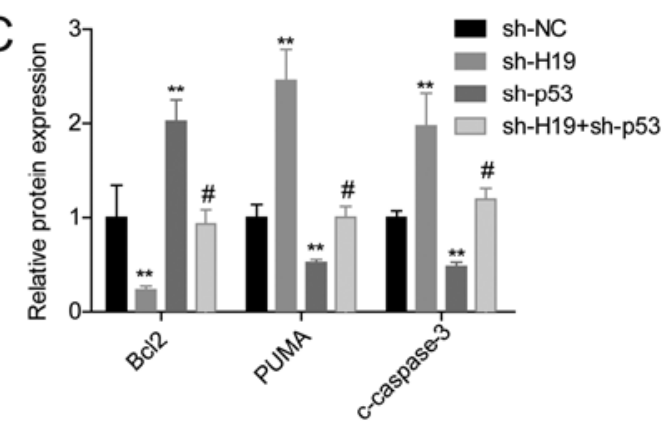

Figure 3. Effects of H19 knockdown on the expression of apoptosis-related proteins. (A) Co-IP assay was performed to evaluate the crosstalk between p53 and Bax proteins after VSMCs were infected with sh-NC or sh-H19. (B and C) The effects of H19/p53 on the expression levels of Bcl-2, PUMA and c-caspase3 were determined by western blotting. ${ }^{* *} \mathrm{P}<0.01$ vs. sh-NC; ${ }^{*} \mathrm{P}<0.05$ vs. sh-H19. c-, cleaved; IP, immunoprecipitation; NC, negative control; PUMA, p53 upregulated modulator of apoptosis; sh, short hairpin RNA; VSMCs, vascular smooth muscle cells.

A
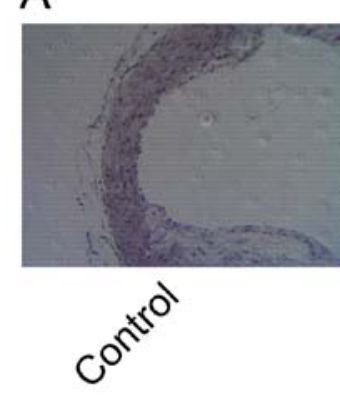

B
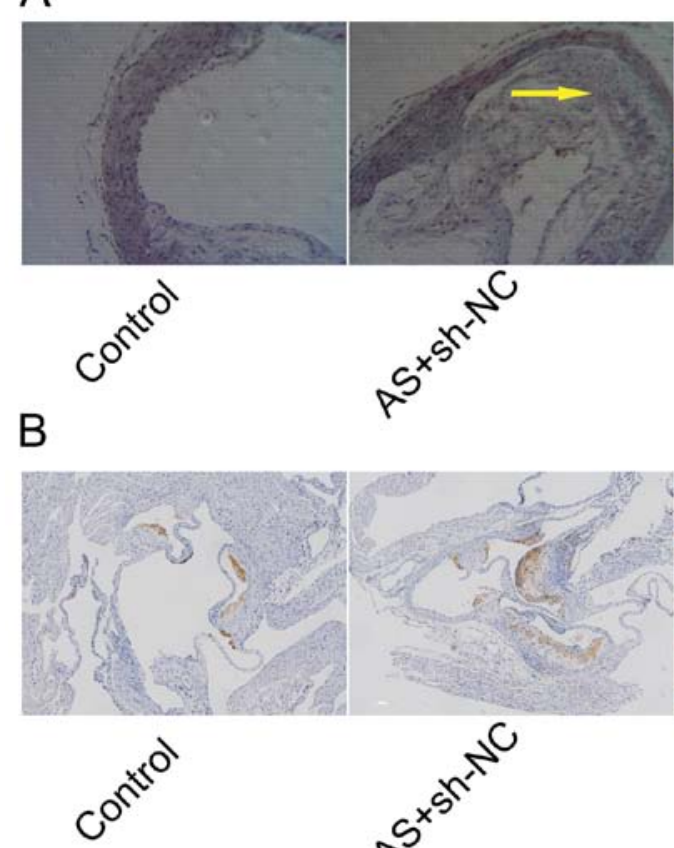
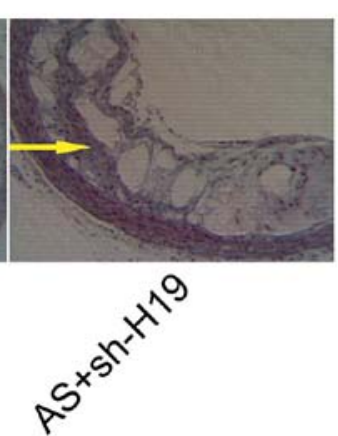

$p$
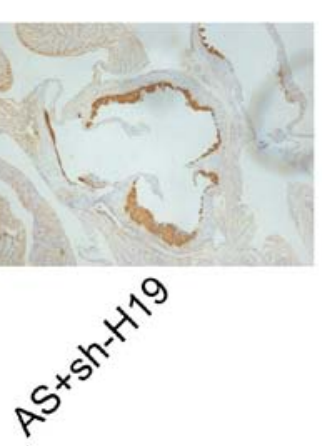
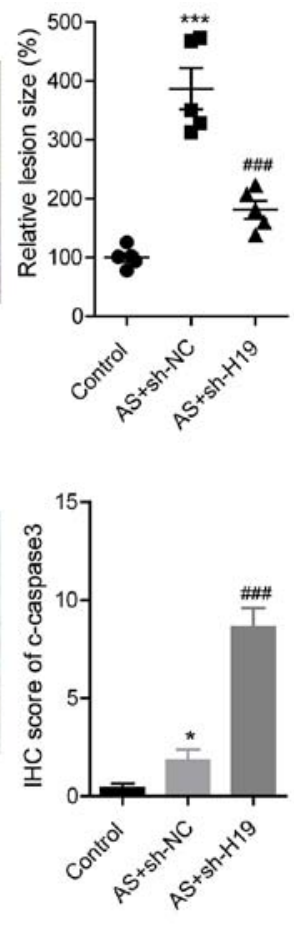

Figure 4. Knockdown of H19 alleviates AS in vivo. (A) Hematoxylin and eosin staining analysis of the lesions in mice aortic roots from different groups. Arrows indicate the atherosclerotic lesions. Magnification, x200. (B) C-caspase 3 expression in atherosclerotic plaque tissues of mice from control, AS + sh-NC or AS + sh-H19 group was analyzed by IHC. Magnification, x100. ${ }^{*} \mathrm{P}<0.05$ and ${ }^{* * * *} \mathrm{P}<0.001$ vs. Control; ${ }^{\# \#} \mathrm{P}<0.001$ vs. AS + sh-NC. AS, atherosclerosis; c-, cleaved; IHC, immunohistochemistry; NC, negative control; sh, short hairpin RNA.

tissues, compared with the AS + sh-NC group (Fig. 4B). These findings suggested that knockdown of $\mathrm{H} 19$ could alleviate high-fat diet-induced plaque formation in $\mathrm{ApoE}^{-/-}$mice.

Knockdown of H19 enhances the role of simvastatin in alleviating AS. Finally, the effects of H19 downregulation on the progression of AS under simvastatin administration was explored in vivo. Compared with the sh-NC group, VSMC apoptosis was significantly increased in both the sh-H19 and sh-NC + simvastatin groups (Fig. 5A and B). In addition, the apoptotic rate of VSMCs in the sh- $\mathrm{H} 19+$ simvastatin group was significantly higher compared with the sh-NC + simvastatin group (Fig. 5A and B). Similarly, the in vivo experiments demonstrated that simvastatin treatment significantly reduced the lesion size in the aortic root in the AS + sh-NC + simvastatin group compared with the AS group, and this effect was significantly enhanced when H19 was downregulated in the AS + sh-H19 + simvastatin group (Fig. 5C). In addition, c-caspase 3 expression was increased in mice atherosclerotic plaque tissues in the AS + simvastatin + sh-H19 group compared with the AS + simvastatin group (Fig. 5D and E), as well as increased expression levels of Bax/Bcl-2 and PUMA (Fig. 5E). These results indicated that $\mathrm{H} 19$ downregulation may cooperate with simvastatin to promote VSMCs apoptosis, thereby alleviating AS.

\section{Discussion}

AS is a clinical symptom that can induce narrowing inside the artery interior due to plaque accumulation. Several 
A
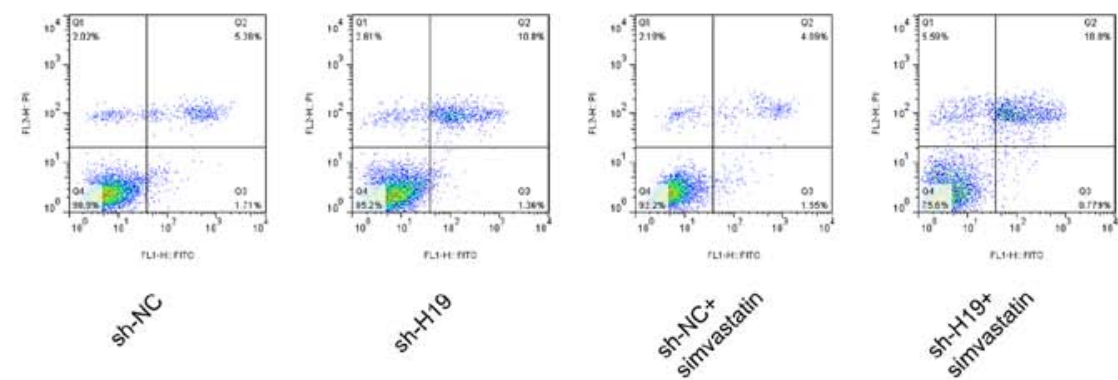

C

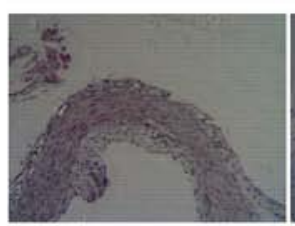

$c^{0 \times 0}$

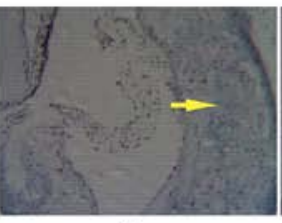

$p^{5}$

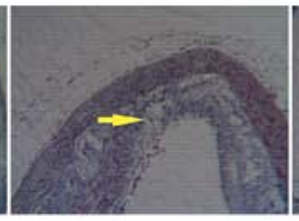

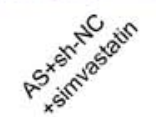

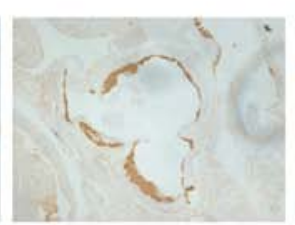

E

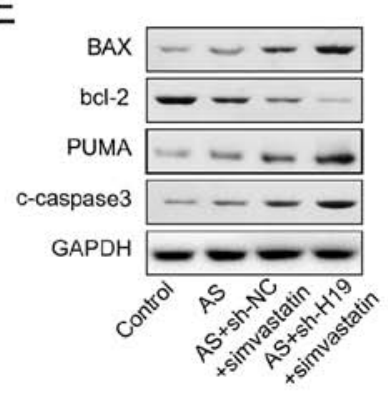

$p^{5}$
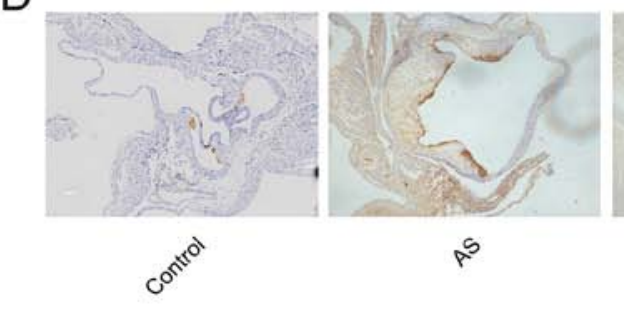

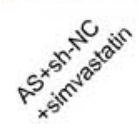

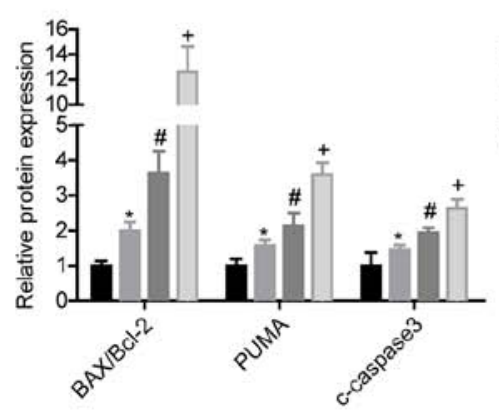
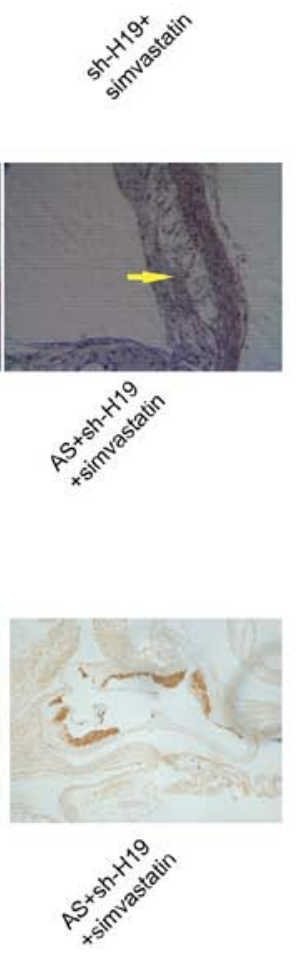
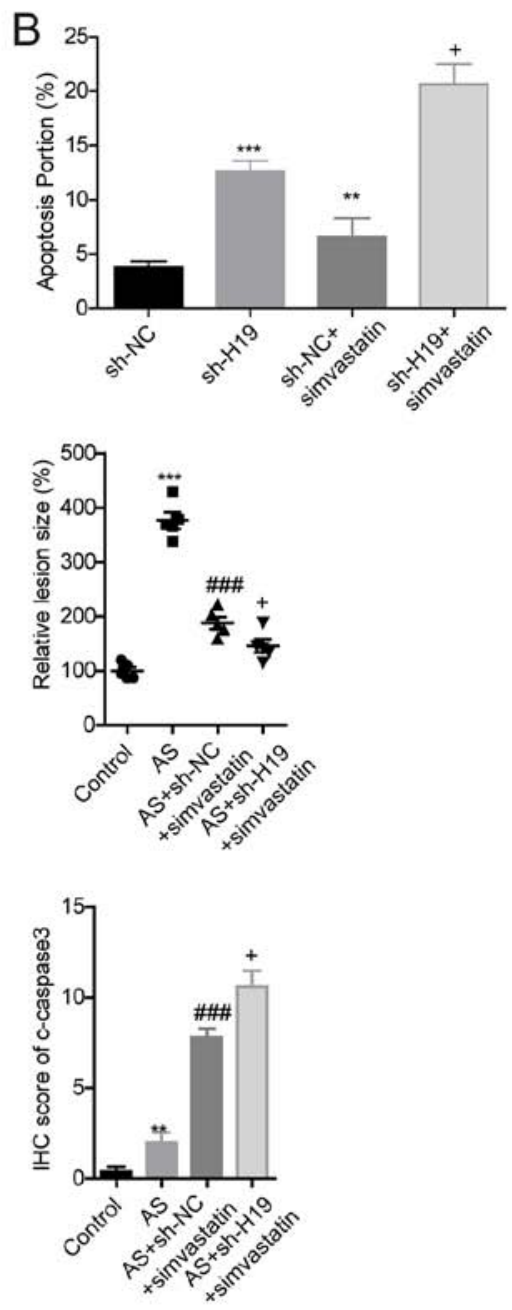

control

AS

- AS+simvastatin

$\square \mathrm{AS}+\mathrm{sh}-\mathrm{H} 19+$ simvastatin

Figure 5. H19 downregulation cooperates with simvastatin to alleviate AS. (A and B) Apoptosis was detected with flow cytometry after VSMCs were infected with sh-NC, sh-H19, sh-NC + simvastatin or sh-H19 + simvastatin. ${ }^{* *} \mathrm{P}<0.01,{ }^{* * *} \mathrm{P}<0.001$ vs. sh-NC; ${ }^{+} \mathrm{P}<0.05$ vs. sh-NC + simvastatin. $(\mathrm{C}) \mathrm{Hematoxylin}$ and eosin staining analysis of the lesions in mice aortic roots from different groups. Arrows indicate the atherosclerotic lesions. Magnification, $\mathrm{x} 200$. (D) C-caspase3 expression levels in the atherosclerotic plaque tissues from mice in control, AS + sh-NC, AS + sh-NC + simvastatin or AS + sh-H19 + simvastatin groups were analyzed by IHC. Magnification, x100. (E) Western blot analysis of the expression levels of Bcl2, PUMA, c-caspase3 and Bax in atherosclerotic plaque tissues of mice from control, AS, AS + simvastatin or AS + simvastatin + sh-H19 groups. ${ }^{*} \mathrm{P}<0.05$ and ${ }^{* * *} \mathrm{P}<0.01,{ }^{* * * *} \mathrm{P}<0.001$ vs. Control; ${ }^{\#} \mathrm{P}<0.05$ and ${ }^{\# \# \#} \mathrm{P}<0.001$ vs. AS; ${ }^{+} \mathrm{P}<0.05$ vs. AS + sh-NC + simvastatin. AS, atherosclerosis; c-, cleaved; IHC, immunohistochemistry; NC, negative control; sh, short hairpin RNA; PUMA, p53 upregulated modulator of apoptosis; VSMCs, vascular smooth muscle cells.

risk factors, including excessive drinking, unbalanced diet, smoking and obesity, may facilitate the progression of AS (2). The dysfunction of VSMCs serves a vital role in the formation and eventual rupture of atherosclerotic plaques due to their hyperproliferative properties (26). The excessive proliferation of VSMCs can trigger plaque formation, which accounts for an important step in the initiation of AS, indicating that effective control of VSMC proliferation may be a promising method for AS prevention and treatment (27). However,
VSMC apoptosis in advanced plaques increase plaque vulnerability, stenosis and medial degeneration $(28,29)$. Collectively, these findings indicate that VSMC apoptosis and proliferation serve dual roles in the pathogenesis of AS. The present study demonstrated that knockdown of lncRNA H19 could inhibit plaque formation through inducing the apoptosis and repressing the proliferation of VSMCs partly in a p53-dependent way, indicating a protective role of H19 downregulation in AS. 
Recently, studies have reported that lncRNAs are strongly implicated in the pathogenesis of AS. For instance, Chen et al (30) found that tanshinol administration could reduce the aortic atherosclerotic lesion area by downregulating lncRNA TUG1. Yao et al (31) demonstrated that lncRNA ENST00113 significantly promotes the proliferation and migration of VSMCs and HUVECs. 1ncRNA H19 was confirmed to be overexpressed in patients with AS, and H19 overexpression significantly promoted the proliferation and repressed the apoptosis of HUVECs and VSMCs in vitro (15). Consistently, the present study clarified that H19 was highly expressed in AS mice, and H19 knockdown significantly inhibited the progression of AS by inducing the apoptosis of VSMCs.

p53 serves an important role in the pathogenesis of AS. For example, Guevara et al (16) reported that deficiency of p53 in $\mathrm{ApoE}^{-/-}$mice accelerated the formation of aortic plaque and increased cell proliferation in the atherosclerotic lesions. van Vlijmen et al (18) demonstrated that p53 expression was increased in human atherosclerotic plaques, such as VSMCs and macrophages, and depletion of it in macrophages can accelerate AS progression in $\mathrm{ApoE}^{-/-}$mice. Wassmann et al (32) reported that upregulation of p53 induced by Krüppel-like factors (such as Krüppel-like Factor 4) can significantly promote VSMCs apoptosis. These data demonstrated that the downregulation of p53 may promote the progression of AS through repressing VSMC apoptosis. The present study demonstrated that knockdown of H19 could increase p53 expression and increase its interaction with Bax, an apoptotic marker. In addition, it was observed that the increase in cell apoptosis caused by H19 downregulation was rescued when p53 was downregulated in VSMCs, which suggested that H19 may promote the apoptosis of VSMCs partly in a p53-dependent manner. This result was congruent to a previous study by Wu et al (21), which demonstrated that upregulation of p53 induces an increase in VSMC apoptosis.

Simvastatin, a cholesterol-lowering drug, is widely used as an atheroprotective drug mainly due to its inhibitory role in cholesterol syntheses $(33,34)$. Increasing evidence has demonstrated that simvastatin stimulation can inhibit the proliferation of VSMCs $(35,36)$. Notably, Chan et al (36) found that p53 upregulation is strongly implicated in simvastatin-mediated VSMC growth repression. Based on this, the present study used simvastatin as a drug for AS treatment in vivo and in vitro to further reveal the roles of H19 in AS progression. The results demonstrated that knockdown of $\mathrm{H} 19$ enhanced the role of simvastatin in promoting VSMC apoptosis and increasing c-caspase 3 expression in atherosclerotic plaque tissues. In conclusion, the present study identified that the knockdown of lncRNA H19 may inhibit AS progression by inducing VSMC apoptosis in a p53-dependent manner.

\section{Acknowledgements}

Not applicable.

\section{Funding}

No funding was received.

\section{Availability of data and materials}

All data generated or analyzed during this study are included in this published article.

\section{Authors' contributions}

$\mathrm{KC}$ conceived the study and revised the manuscript. HS and QJ performed all the experiments, performed data analysis and were major contributors to the writing of the manuscript. LS performed some of the experiments. All authors read and approved the final manuscript.

\section{Ethics approval and consent to participate}

Animal experiment was approved by The Committee of Jining First People's Hospital (Jining, China) and were performed in accordance with the National Institutes of Health Guide for the Care and Use of Laboratory Animals.

\section{Patient consent for publication}

Not applicable.

\section{Competing interests}

The authors declare that they have no competing interests.

\section{References}

1. Schaftenaar F, Frodermann V, Kuiper J and Lutgens E: Atherosclerosis: The interplay between lipids and immune cells. Curr Opin Lipdol 27: 209-215, 2016.

2. Weber $\mathrm{C}$ and Noels $\mathrm{H}$ : Atherosclerosis: Current pathogenesis and therapeutic options. Nat Med 17: 1410-1422, 2011.

3. Moore KJ, Sheedy FJ and Fisher EA: Macrophages in atherosclerosis: A dynamic balance. Nat Rev Immunol 13: 709-721, 2013.

4. Galkina E and Ley K: Immune and inflammatory mechanisms of atherosclerosis. Ann Rev Immunol 27: 165-197, 2009.

5. Leischik R, Foshag P, Straub M, Garg P, Dworrak B, Littwitz H, Lazic JS and Horlitz M: Physical activity, cardiorespiratory fitness and carotid intima thickness: Sedentary occupation as risk factor for atherosclerosis and obesity. Eur Rev Med Pharmacol Sci 19: 3157-3168, 2015

6. Bennett MR, Sinha S and Owens GK: Vascular smooth muscle cells in atherosclerosis. Circ Res 118: 692-702, 2016.

7. Kapranov P, Willingham AT and Gingeras TR: Genome-wide transcription and the implications for genomic organization. Nat Rev Genet 8: 413-423, 2007.

8. Yang JX, Rastetter RH and Wilhelm D: Non-coding RNAs: An introduction. Adv Exp Med Biol 886: 13-32, 2016.

9. Rinn JL and Chang HY: Genome regulation by long noncoding RNAs. Ann Rev Biochem 81: 145-166, 2012.

10. Huarte M: The emerging role of lncRNAs in cancer. Nat Med 21: 1253-1261, 2015.

11. Lorenzen JM and Thum T: Long noncoding RNAs in kidney and cardiovascular diseases. Nat Rev Nephrol 12: 360-373, 2016.

12. Zhou T, Ding JW, Wang XA and Zheng XX: Long noncoding RNAs and atherosclerosis. Atherosclerosis 248: 51-61, 2016.

13. Jian L, Jian D, Chen Q and Zhang L: Long noncoding RNAs in atherosclerosis. J Atheroscler Thromb 23: 376-384, 2016.

14. Kallen AN, Zhou XB, Xu J, Qiao C, Ma J, Yan L, Lu L, Liu C, Yi JS, Zhang H, et al: The imprinted H19 lncRNA antagonizes let-7 microRNAs. Mol Cell 52: 101-112, 2013.

15. Pan JX: LncRNA H19 promotes atherosclerosis by regulating MAPK and NF-kB signaling pathway. Eur Rev Med Pharmacol Sci 21: 322-328, 2017.

16. Guevara NV, Kim HS, Antonova EI and Chan L: The absence of p53 accelerates atherosclerosis by increasing cell proliferation in vivo. Nat Med 5: 335-339, 1999. 
17. Merched AJ, Williams E and Chan L: Macrophage-specific p53 expression plays a crucial role in atherosclerosis development and plaque remodeling. Arterioscler Thromb Vasc Biol 23: $1608-1614,2003$

18. van Vlijmen BJ, Gerritsen G, Franken AL, Boesten LS, Kockx MM, Gijbels MJ, Vierboom MP, van Eck M, van De Water B, van Berkel TJ and Havekes LM: Macrophage p53 deficiency leads to enhanced atherosclerosis in APOE*3-Leiden transgenic mice. Circ Res 88: 780-786, 2001.

19. Mercer J and Bennett M: The role of p53 in atherosclerosis. Cell Cycle 5: 1907-1909, 2006.

20. Mercer J, Figg N, Stoneman V, Braganza D and Bennett MR: Endogenous p53 protects vascular smooth muscle cells from apoptosis and reduces atherosclerosis in ApoE knockout mice. Circ Res 96: 667-674, 2005.

21. Wu G, Cai J, Han Y, Chen J, Huang ZP, Chen C, Cai Y, Huang H, Yang Y, Liu Y, et al: LincRNA-p21 regulates neointima formation, vascular smooth muscle cell proliferation, apoptosis, and atherosclerosis by enhancing p53 activity. Circulation 130: 1452-1465, 2014.

22. Ma L, Qian L, Ying Q, Zhang Y, Zhou C and Wu G: I4, a synthetic anti-diabetes agent, attenuates atherosclerosis through its lipid-lowering, anti-inflammatory and anti-apoptosis properties. Mol Cell Endocrinol 440: 80-92, 2017.

23. Yin M, Liu Q, Yu L, Yang Y, Lu M, Wang H, Luo D, Rong X, Tang F and Guo J: Downregulations of CD36 and calpain-1, inflammation, and atherosclerosis by simvastatin in apolipoprotein E knockout mice. J Vasc Res 54: 123-130, 2017.

24. Xin B, He X, Wang J, Cai J, Wei W, Zhang T and Shen X: Nerve growth factor regulates CD133 function to promote tumor cell migration and invasion via activating ERK1/2 signaling in pancreatic cancer. Pancreatology 16: 1005-1014, 2016.

25. Livak KJ and Schmittgen TD: Analysis of relative gene expression data using real-time quantitative PCR and the 2(-Delta Delta C(T)) method. Methods 25: 402-408, 2001

26. Gorenne I, Kumar S, Gray K, Figg N, Yu H, Mercer J and Bennett M: Vascular smooth muscle cell sirtuin 1 protects against DNA damage and inhibits atherosclerosis. Circulation 127 386-396, 2013

27. Rudijanto A: The role of vascular smooth muscle cells on the pathogenesis of atherosclerosis. Acta Med Indones 39: 86-93, 2007.

28. Clarke MC, Figg N, Maguire JJ, Davenport AP, Goddard M, Littlewood TD and Bennett MR: Apoptosis of vascular smooth muscle cells induces features of plaque vulnerability in atherosclerosis. Nat Med 12: 1075-1080, 2006.
29. Clarke MC, Littlewood TD, Figg N, Maguire JJ, Davenport AP, Goddard M and Bennett MR: Chronic apoptosis of vascular smooth muscle cells accelerates atherosclerosis and promotes calcification and medial degeneration. Circ Res 102: 1529-1538, 2008.

30. Chen C, Cheng G, Yang X, Li C, Shi R and Zhao N: Tanshinol suppresses endothelial cells apoptosis in mice with atherosclerosis via lncRNA TUG1 up-regulating the expression of miR-26a. Am J Transl Res 8: 2981-2991, 2016.

31. Yao X, Yan C, Zhang L, Li Y and Wan Q: LncRNA ENST00113 promotes proliferation, survival, and migration by activating $\mathrm{PI} 3 \mathrm{~K} / \mathrm{Akt} / \mathrm{mTOR}$ signaling pathway in atherosclerosis. Medicine (Baltimore) 97: e0473, 2018.

32. Wassmann S, Wassmann K, Jung A, Velten M, Knuefermann P, Petoumenos V, Becher U, Werner C, Mueller C and Nickenig G: Induction of p53 by GKLF is essential for inhibition of proliferation of vascular smooth muscle cells. J Mol Cell Cardiol 43: 301-307, 2007.

33. Song G, Liu J, Zhao Z, Yu Y, Tian H, Yao S, Li G and Qin S Simvastatin reduces atherogenesis and promotes the expression of hepatic genes associated with reverse cholesterol transport in apoE-knockout mice fed high-fat diet. Lipids Health Dis 10: 8 , 2011.

34. Sparrow CP, Burton CA, Hernandez M, Mundt S, Hassing H, Patel S, Rosa R, Hermanowski-Vosatka A, Wang PR, Zhang D, et al: Simvastatin has anti-inflammatory and antiatherosclerotic activities independent of plasma cholesterol lowering. Arterioscler Thromb Vasc Biol 21: 115-121, 2001.

35. Zhang Z, Zhang M, Li Y, Liu S, Ping S, Wang J, Ning F, Xie F and Li C: Simvastatin inhibits the additive activation of ERK1/2 and proliferation of rat vascular smooth muscle cells induced by combined mechanical stress and oxLDL through LOX-1 pathway. Cell Signal 25: 332-340, 2013.

36. Chan KC, Wang CJ, Ho HH, Chen HM and Huang $\mathrm{CN}$ Simvastatin inhibits cell cycle progression in glucose-stimulated proliferation of aortic vascular smooth muscle cells by up-regulating cyclin dependent kinase inhibitors and p53. Pharmacol Res 58: 247-256, 2008.

(i) $\Theta$ This work is licensed under a Creative Commons Attribution-NonCommercial-NoDerivatives 4.0 International (CC BY-NC-ND 4.0) License. 